\title{
Empirical Modelling and Simulation of Transmission Loss between Wireless Sensor Nodes in Gas Turbine Engines
}

\author{
Konstantinos Sasloglou*, Ian A. Glover *, Partha Dutta ${ }^{\dagger}$, Robert Atkinson * , Ivan Andonovic * and Griogair Whyte ${ }^{\ddagger}$ \\ * Department of Electronic \& Electrical Engineering \\ University of Strathclyde, Glasgow, United Kingdom \\ Email: $\{$ ksasloglou, ian.glover, r.atkinson, i.andonovic\}@eee.strath.ac.uk \\ ${ }^{\dagger}$ Information Engineering Group, Strategic Research Centre, Rolls-Royce, Derby, United Kingdom \\ Email: partha.s.dutta@ rolls-royce.com \\ ‡Electronic Design Centre, Department of Electronic \& Electrical Engineering, \\ The University of Glasgow, Glasgow, United Kingdom \\ E-mail: G.Whyte@elec.gla.ac.uk
}

\begin{abstract}
Transmission loss measurements between a grid of hypothetical WSN node locations on the surface of a gas turbine engine are reported for eight frequencies at $1 \mathrm{GHz}$ intervals in the frequency range 3.0 to 11.0 GHz. An empirical transmission loss model is derived from the measurements. The model is incorporated into an existing system channel model implemented using Simulink as part of a wider project concerning the development of WSNs for the testing and condition monitoring of gas turbine engines.
\end{abstract}

Index Terms-wireless sensor networks; antennas; propagation; channel model; gas turbine engine

\section{INTRODUCTION}

Testing of gas turbine engines currently demands the use of an extensive wiring harness to connect sensing nodes to data recording and analysis equipment. The size and complexity of the harness makes setup procedures complex, lengthy and expensive. It also makes changes cumbersome and inconvenient which limits flexibility and the opportunity to make ad-hoc changes to later measurements that might be suggested by earlier measurements. The replacement of the fixed wiring harness with a wireless sensor network (WSN) has obvious advantages in this respect. The work reported here addresses the measurement and modelling of the physical channel characteristics that any such WSN would be required to accommodate. It is part of a wider project - Wireless Data Acquisition in Gas Turbine Engines (WIDAGATE) which is being undertaken by a consortium comprising RollsRoyce, Selex, University College London and the University of Strathclyde.

\section{Methodology}

Transmission loss measurements have been made between pairs of points forming a grid over the cylindrical surface of a Gnome gas turbine engine, Figure 1.

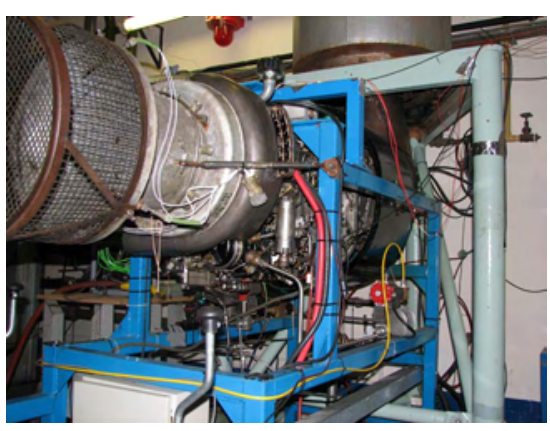

Fig. 1. Gnome engine.

The Gnome engine is approximately cylindrical. Its length is $78 \mathrm{~cm}$ and its mean radius is $18 \mathrm{~cm}$. The steel cradle in which the engine was mounted was shrouded, as far as possible, using flexible microwave absorber to reduce unwanted electromagnetic scattering. The measurements have been made using an Agilent N5230A network analyser to obtain the frequency response over the range $3.0-11.0 \mathrm{GHz}$ between a pair of ultra-wideband (UWB), omni-directional, antennas, Figure 2.

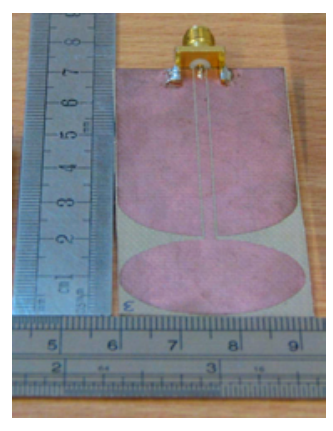

Fig. 2. UWB antenna. 
The measurements have been made both in the absence, Figure 3, and presence, Figure 4, of an engine cowling. These measurements are referred to here as set A and B respectively.

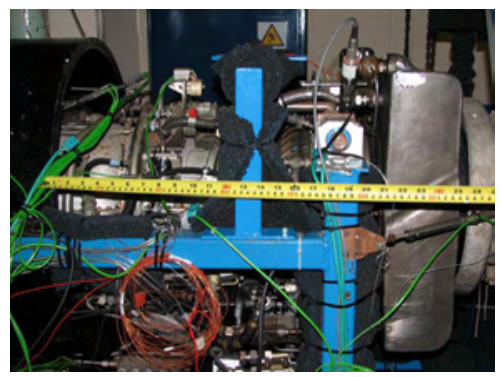

Fig. 3. Engine without cowling.

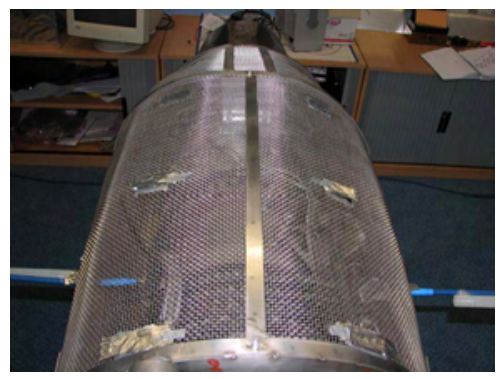

Fig. 4. Engine with cowling.

The engine cowling was manufactured by SCITEK Consultants Ltd. from stainless steel mesh, Figure 4.

These measurement databases have been used to derive an empirical narrowband transmission gain model for frequencies within the UWB band $(3.1-10.6 \mathrm{GHz})$.

Figure 5 shows the measured return loss of one of the (identical pair of) antennas. $\left(S_{11}\right)$.

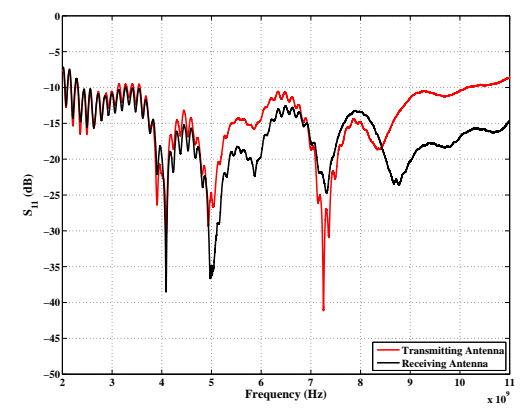

Fig. 5. Antenna return loss $\left(S_{11}\right)$.

Each antenna was connected to the network analyser using $10 \mathrm{~m}$ of low-loss, high quality coaxial cable.

The antennas were end-mounted on dielectric rods to keep the metallic structure of the antenna positioners as far as possible from the measurement volume. Like the engine cradle, the antenna positioners were shrouded in microwave absorber to further reduce the effect of unwanted scattering on the measurements.

The resolution of the measured discrete frequency response was $537.46 \mathrm{kHz}$.

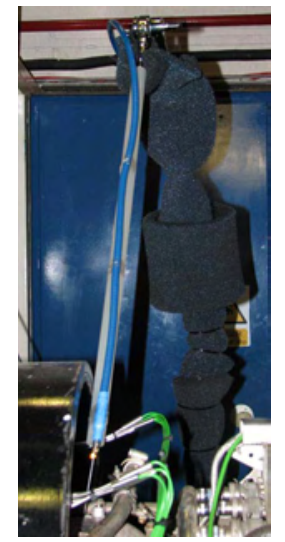

Fig. 6. Antenna positioners.

\section{A. Measurement Set A}

The measurements of transmission loss for the engine without cowling (measurement set A) were made in the Gnome Test Laboratory at Rolls-Royce in Derby, UK. Figure 7 shows the nominal topology of the measurement points (representing hypothetical nodes). Nominal node locations are assumed at $0^{\circ}, 60^{\circ}, 120^{\circ}$, and $180^{\circ}$ on the engine circumference in each of five planes resulting in 20 node locations and 190 potential measurements. (Points at $240^{\circ}$ and $300^{\circ}$ are not represented since these give rise to redundant link geometries.) The planes, which were perpendicular to the axis of the engine, are separated by $28 \mathrm{~cm}, 24 \mathrm{~cm}, 6 \mathrm{~cm}$ and $20 \mathrm{~cm}$. Two of the 190 measurements were lost due to an error when saving the relevant files.

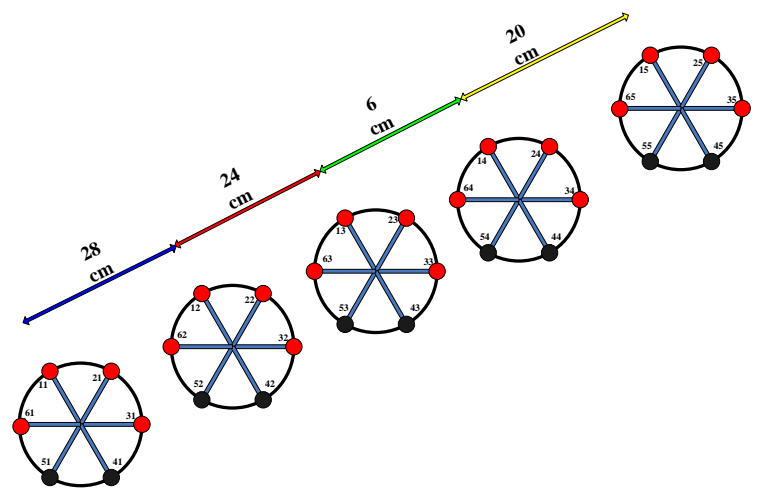

Fig. 7. Nominal topology of measurement points without cowling (set A).

\section{B. Measurement Set B}

The measurements of transmission loss for the engine with cowling (measurement set B) were made in the University of Strathclyde Wireless Communications Laboratory.

The engine used was of the same (Gnome) type as that used for measurement set A but was not the same engine. The surface detail of the engine was therefore different. (Since the surface detail represents an essentially random distribution of scatterers the use of two engines is not thought to materially reduce the usefulness of the resulting statistical model.) Figure 
8 shows the nominal topology of the measurement points. Similarly to the measurements in set A, nominal node locations are assumed at $0^{\circ}, 60^{\circ}, 120^{\circ}$, and $180^{\circ}$ on the engine circumference in each of three planes resulting in 12 node locations and 66 potential measurements. The planes, which were perpendicular to the axis of the engine, are separated by $25 \mathrm{~cm}$ and $26 \mathrm{~cm}$.

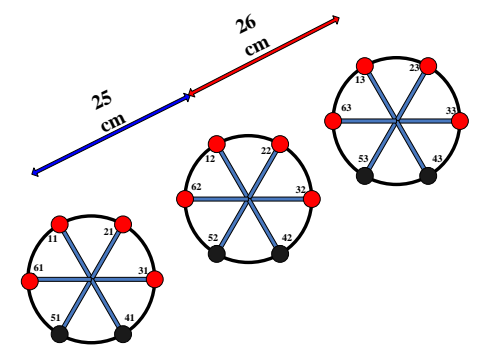

Fig. 8. Nominal topology of measurement points with cowling (set B).

Six of the 66 measurements were lost due to measurement error or corruption of data during saving.

\section{TRANSMISSION GAIN MODEL}

An empirical model of transmission gain (i.e. ratio of received to transmitted power) has been fitted to the measurement data. In order that the model might be applied to engines of similar type but different size the model is regressed on path length, $s$, and path curvature, $\kappa$. (Curvature is the reciprocal of radius of curvature.) Figure 9 defines of these parameters.

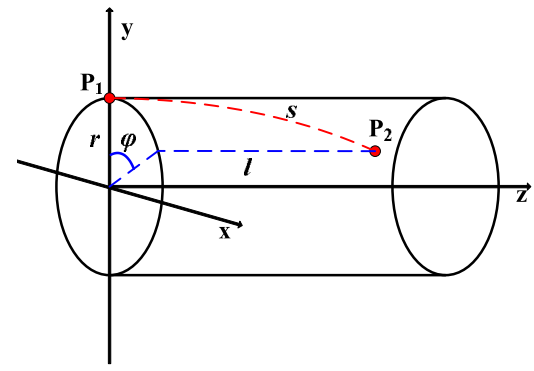

Fig. 9. Path geometry.

The path length of each measurement is that of a curved arc connecting transmitter $\left(P_{1}\right)$ and receiver $\left(P_{2}\right)$. Path length $s$ is related to the lateral displacement between planes containing the measurement points, $l$, the angular separation, $\phi$, between measurement points projected into a single plane and the engine radius, $r$, by:

$$
s=\sqrt{(r \phi)^{2}+l^{2}}
$$

Similarly, path curvature is given by:

$$
\kappa=\frac{r}{r^{2}+\left(\frac{l}{\phi}\right)^{2}}
$$

It is assumed that path length and path curvature are sufficient to predict transmission loss. In principal there are many distinguishable paths with the same length and curvature, but different torsion but, for pragmatic engineering purposes, torsion is ignored.

A first-degree polynomial, $A s+B \kappa+C \bar{G}_{T}+D=0$, where $\bar{G}_{T}$ is mean transmission gain in $\mathrm{dB}(<0)$, which best fits the empirical data in each $1 \mathrm{GHz}$ frequency band has been obtained. Examples are shown in Figures 10 and 11, respectively, for measurements without and with a cowling for the $6-7 \mathrm{GHz}$ band.

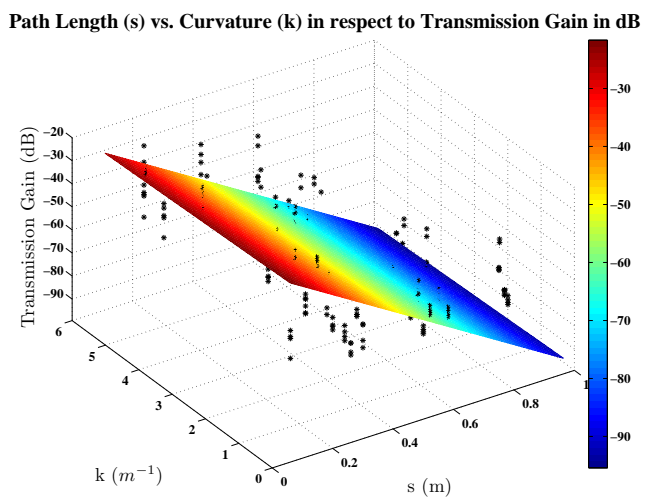

Fig. 10. Example of best-fit transmission gain model for measurement set A $(6 \mathrm{GHz}-7 \mathrm{GHz})$.

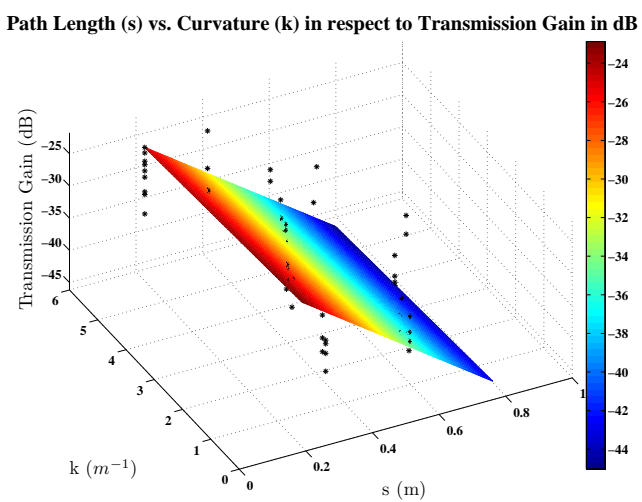

Fig. 11. Example of best-fit transmission gain model for measurement set $\mathrm{B}$ $(6 \mathrm{GHz}-7 \mathrm{GHz})$.

Figures 12 and 13 show the projection of Figures 1011 , respectively, chosen to illustrate the influence of path length.

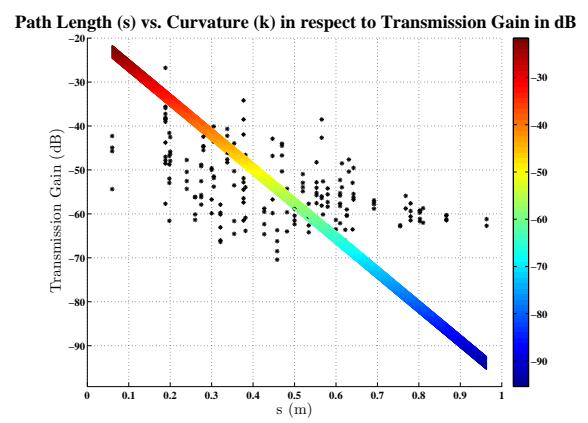

Fig. 12. Two dimensional projection of data (set 1) illustrating transmission gain versus path length $(6 \mathrm{GHz}-7 \mathrm{GHz})$. 


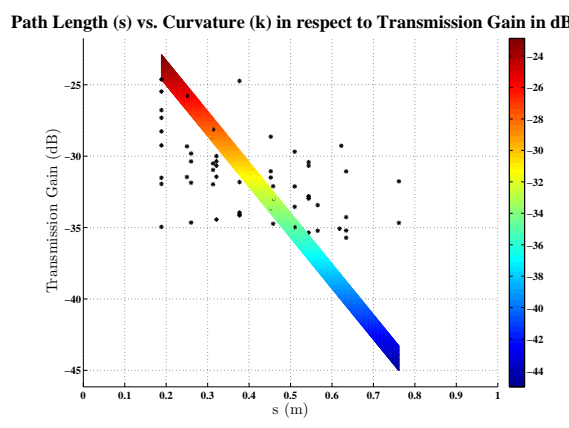

Fig. 13. Two dimensional projection of data (set 2) illustrating transmission gain versus path length $(6 \mathrm{GHz}-7 \mathrm{GHz})$.

Table I shows the best-fit polynomial coefficients derived for each measurement set at each frequency.

\begin{tabular}{|c|c|c|c|c|c|}
\hline & & \multicolumn{4}{|c|}{ Polynomial Coefficients } \\
\hline & $f$ & $\mathrm{~A}$ & $\mathrm{~B}$ & $\mathrm{C}$ & $\mathrm{D}$ \\
\hline \hline Meas. Set A & $3.0-4.0$ & -0.9997 & -0.0203 & -0.0139 & -0.1972 \\
\hline & $4.0-5.0$ & -0.9998 & -0.0125 & -0.0134 & -0.1925 \\
\hline & $5.0-6.0$ & 0.9999 & 0.0049 & 0.0131 & 0.2044 \\
\hline & $6.0-7.0$ & 0.9999 & 0.0066 & 0.0127 & 0.2161 \\
\hline & $7.0-8.0$ & -0.9998 & -0.0080 & -0.0139 & -0.2953 \\
\hline & $8.0-9.0$ & 0.9998 & 0.0073 & 0.0157 & 0.4091 \\
\hline & $9.0-10.0$ & 0.9998 & 0.0039 & 0.0160 & 0.4500 \\
\hline & $10.0-11.0$ & 0.9998 & 0.0037 & 0.0163 & 0.4846 \\
\hline \hline Meas. Set B & $3.0-4.0$ & -0.9994 & -0.0084 & -0.0327 & -0.4314 \\
\hline & $4.0-5.0$ & -0.9997 & -0.0029 & -0.0202 & -0.1315 \\
\hline & $5.0-6.0$ & -0.9996 & 0.0018 & -0.0258 & -0.3390 \\
\hline & $6.0-7.0$ & 0.9995 & 0.0088 & 0.0280 & 0.4536 \\
\hline & $7.0-8.0$ & -0.9997 & -0.011 & -0.0201 & -0.2219 \\
\hline & $8.0-9.0$ & -0.9997 & -0.0116 & -0.0189 & -0.1825 \\
\hline & $9.0-10.0$ & -0.9997 & -0.0116 & -0.0182 & -0.1842 \\
\hline & $10.0-11.0$ & -0.9998 & -0.0083 & -0.0150 & -0.0947 \\
\hline & & & & & \\
\hline
\end{tabular}

TABLE I

TRANSMISSION GAIN POLYNOMIAL MODEL COEFFICIENTS.

The error between the measured data and the model, i.e.:

$$
\epsilon_{d B}=G_{T, \text { measured }}-G_{T, \text { model }}
$$

has been calculated and itself modelled using the same process as described above, Figure 14 and 15.

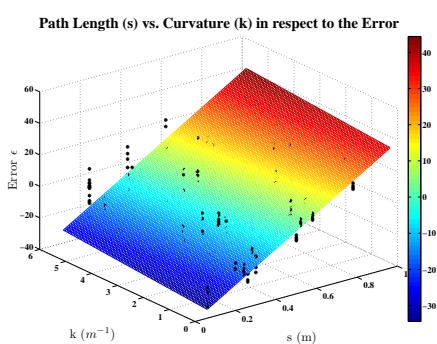

(a)

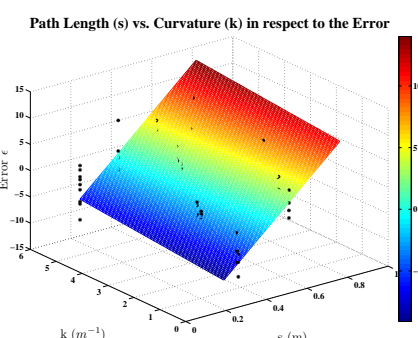

(b)
Fig. 14. Error $(\epsilon)$ between measured data and model: (a) measurement set A, (b) measurement set $\mathrm{B}(6 \mathrm{GHz}-7 \mathrm{GHz})$.

The error as a function of $s$ and $\kappa$ is quantised (four intervals for $s$, three intervals for $(\kappa)$ and a two-dimensional histogram

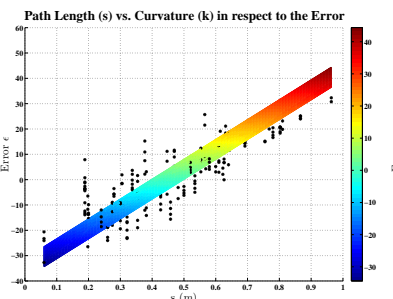

(a)

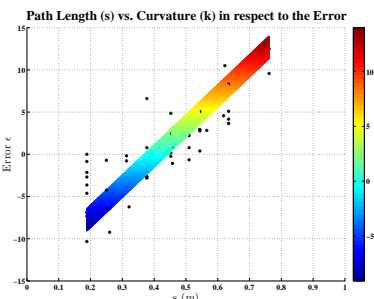

(b)
Fig. 15. Projection of data in Figures 14(a) and (b) illustrating dependence of $\epsilon$ on path length $(6 \mathrm{GHz}-7 \mathrm{GHz})$.

of $\epsilon$ is constructed. The mean and standard deviation of the error for each pair of $s$ and $\kappa$ intervals has been calculated. Figure 16 is an example illustration of the best-fit Gaussian curve (i.e. with identical mean and standard deviation to the error data). Finally, $\mu$ and $\sigma$ are again regressed on $s$ and $\kappa$ using a first degree polynomial surface as before, Figures 17 and 18 .

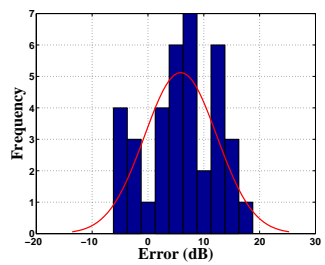

Fig. 16. Error histogram and corresponding Gaussian distribution for a single quantised pair of $s-\kappa$.

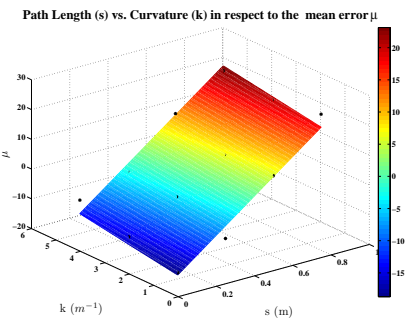

(a)

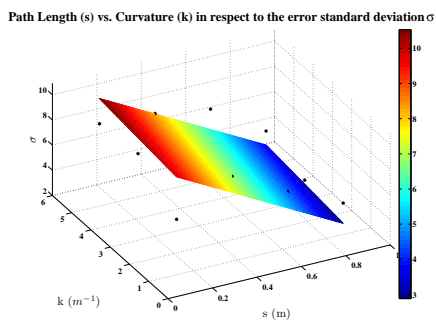

(b)
Fig. 17. $\mu$ versus $s, \kappa$ and $\sigma$ versus $s, \kappa$ for measurement set A $(6 \mathrm{GHz}-7$ $\mathrm{GHz}$ ).

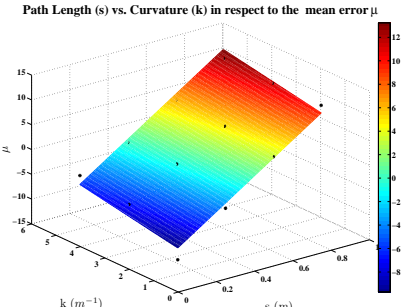

(a)

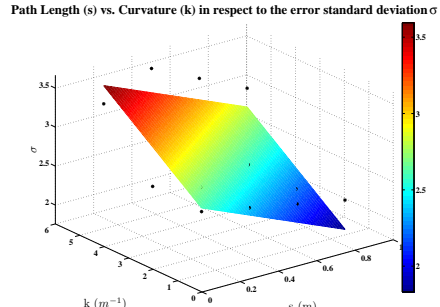

(b)
Fig. 18. $\mu$ versus $s, \kappa$ and $\sigma$ versus $s, \kappa$ for measurement set $\mathrm{B}(6 \mathrm{GHz}-7$ $\mathrm{GHz}$ ).

A system transmission gain model has been implemented using Simulink for application to other parts of the WIDAGATE project. The model includes deterministic and random 
components, i.e. $\bar{G}_{T}(s, \kappa)$, and $e(s, \kappa)$, respectively. $e$ is a Gaussian random variable with mean and standard deviation that depend on $s$ and $\kappa$, i.e.:

$$
G_{T}(s, \kappa)=\bar{G}_{T}(s, \kappa)+e[\mu(s, \kappa), \sigma(s, \kappa)]
$$

A block diagram of the system transmission gain model is shown in Figure 19 in which $g_{i}(t)$ is the transmitted signal.

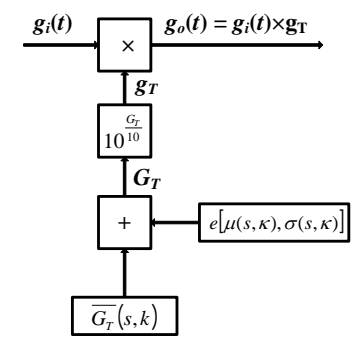

Fig. 19. Transmission gain model.

\section{Interference, Noise ANd Overall Channel MODELS}

An interference model has been constructed using information drawn from [1]. The transmission model, the interference model and an elementary Gaussian noise model [2] (incorporating antenna noise temperature and receiver noise figure [3]) have been integrated into a channel model, Figure 20, implemented in Simulink, Figure 21. The system channel model is currently being incorporated into a WSN system model for the development of a protocol stack optimised for this application.

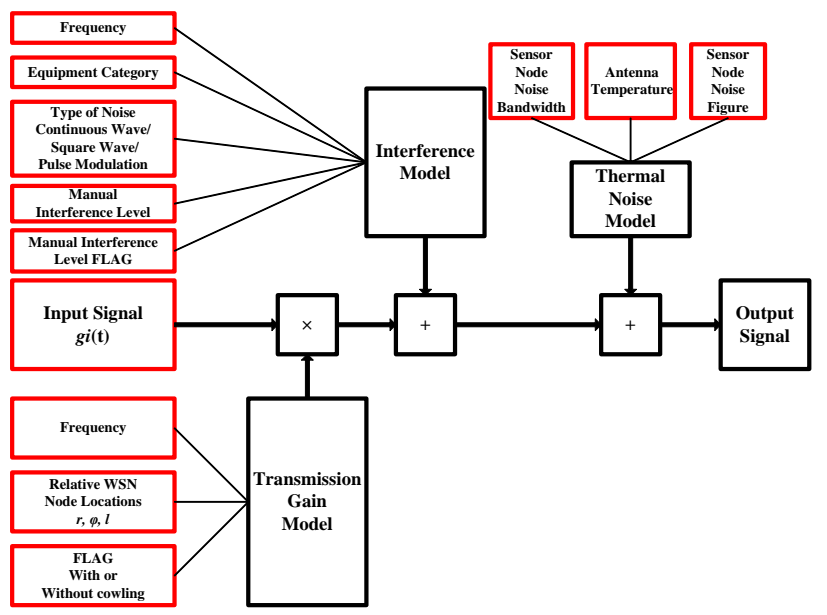

Fig. 20. Overall channel model.

\section{CONCLUSION}

Transmission loss measurements for the application of wireless sensor network technology to gas turbine engine testing have been reported. The measurements cover the UWB frequency band. A simple empirical model comprising bestfit planar regression in path length and path curvature has

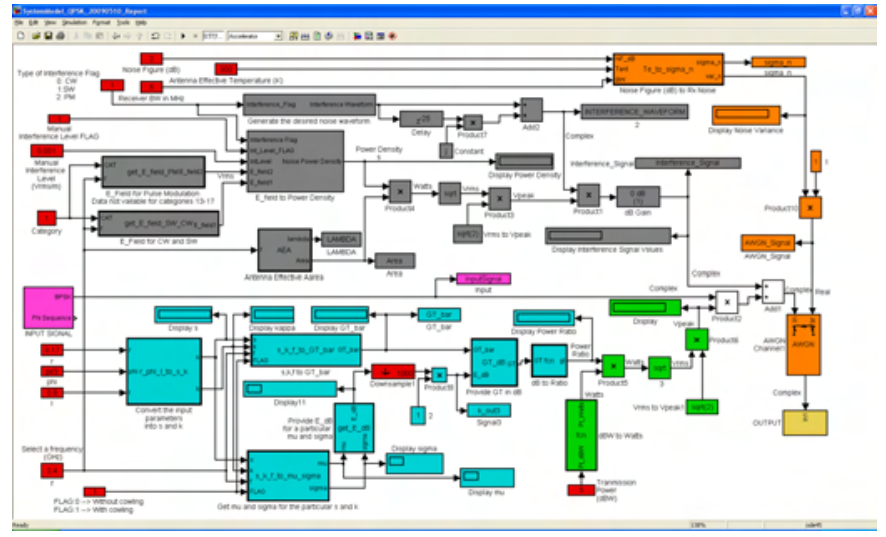

Fig. 21. Simulink implementation of overall channel model.

been derived for each $1 \mathrm{GHz}$ frequency interval within the UWB band. The error between model and measurements as a function of path length and curvature has been characterised. The resulting transmission gain model has been incorporated into a simulator along with an interference and elementary noise model. The simulator is intended for use within a larger project concerned with the investigation of the potential of WSNs for gas turbine engine testing.

\section{ACKNOWLEDGMENT}

This work was supported by the Engineering and Physical Sciences Research Council [grant number TS/G002681/1] as part of the Technology Strategy Board project Gathering Data in Complex Environments [project number: TP11/GCE/6/I/AH135A].

The Technology Strategy Board is a business-led executive non-departmental public body, established by the government. Its role is to promote and support research into, and development and exploitation of, technology and innovation for the benefit of UK business, in order to increase economic growth and improve the quality of life. It is sponsored by the Department for Innovation, Universities and Skills (DIUS). For further information please visit www.innovateuk.org.

The authors acknowledge the contribution of the following collaborative partners and individuals in the shaping and management of the work: Graham B. Hesketh, Armin Stranjak (Rolls-Royce Strategic Research Centre), Yang Yang, Kaan Bur, X. Dai (University College London), Douglas Friedrich, Jason Lepley, Graham Bourdon (Selex Galileo, UK). The support of SCITEK Consultants Ltd. in making measurements at Rolls-Royce's laboratory facilities in Derby is also acknowledged.

\section{REFERENCES}

[1] EUROCAE ED-14E "A Joint EUROCAE RTCA Achievement" [Sections 19-21]. Technical report, The European Organisation for Civil Aviation Equipment, March 2005.

[2] John G. Proakis and Masoud Salehi. Digital Communications. McGrawHill, Fifth edition, 2008.

[3] Ian A. Glover and Peter M. Grant. Digital Communications. Pearson Education Limited, Second edition, 2004. 